\title{
Automatic detection of pneumonia in chest X-rays using Lobe deep residual network
}

\author{
Daniel Kvak ${ }^{1}$ Karolína Kvaková ${ }^{2}$
}

April 2021

\begin{abstract}
:
One of the critical tools for early detection and subsequent evaluation of the incidence of lung diseases is chest radiography. At a time when the speed and reliability of results, especially for COVID-19 positive patients, is important, the development of applications that would facilitate the work of untrained staff involved in the evaluation is also crucial. Our model takes the form of a simple and intuitive application, into which you only need to upload X-rays: tens or hundreds at once. In just a few seconds, the physician will determine the patient's diagnosis, including the percentage accuracy of the estimate. While the original idea was a mere binary classifier that could tell if a patient was suffering from pneumonia or not, in this paper we present a model that distinguishes between a bacterial disease, a viral infection, or a finding caused by COVID-19. The aim of this research is to demonstrate whether pneumonia can be detected or even spatially localized using a uniform, supervised classification.
\end{abstract}

Keywords: automatic detection, chest X-ray, convolutional neural network, COVID-19, deep learning, feature extraction, image classification, pneumonia.

\footnotetext{
${ }^{1}$ Faculty of Arts, Masaryk University, Brno. kvak@mail.muni.cz

${ }^{2}$ Faculty of Science, Masaryk University, Brno.460405@mail.muni.cz
} 


\section{Introduction}

Chest radiography is an important tool for early detection and subsequent verification of lung diseases. These are devices that are easily accessible, and the price of scanning is only in the order of few dollars (Du et al. 2021). However, in the current pandemic situation of COVID19 , we encounter a lack of radiologists and trained staff to analyze the vast number of images taken. The limited availability of high-resolution computed tomography and polymerase chain reaction (RT-PCR) in countries with high patient turnover underlines the importance of chest $\mathrm{X}$-rays as a suitable tool for screening and diagnosis.

To date, the most widely used method for detecting COVID-19 pneumonia is the RT-PCR test, which takes approximately 4 hours to evaluate (Gandhi et al. 2020). With the time needed for collection and transport, we can thus range in the range of days, with not every region having an evaluation center; not to mention the price of up to several hundred dollars (Du et al. 2021). Reliability converging to $100 \%$ does not appear until the third repetition (Gandhi et al. 2020). Another method is represented by antigen tests, which offer cheaper and faster detection, but a positive result might be shown only if the individual is at the stage of greatest risk of transmission. An increasingly common procedure is the detection of antibodies, but these are found in the body one to three weeks after the infection (Mina et al. 2021). The aim of this research is to present a deep learning approach that would eliminate the time and resources needed to develop new technologies and related algorithms. The results presented in this text suggest that the Lobe application based on the deep residual network ResNet-50 could represent an optimal solution for the detection of X-ray findings of pneumonia with a success rate of up to $99 \%$.

\section{Applied deep neural network architecture}

It is the unpretentious and user-friendly form of the used model that is the decisive factor why we believe that the application of pulmonary findings detection mentioned below has the potential for use in practice. Despite the simple design, the program hides one of the most modern solutions for pattern recognition in image data (Papers with Code 2021). The ResNet50 deep neural network, first presented by He et al. (2015), introduces state-of-the-art computer vision technology. This architecture uses a method of extracting features from image files, thanks to which the network learns according to which perceptions it can sort individual images into different classes. Because the findings falling under the same class show similar deviations, 
the application uses a prediction capability, which then divides the new images into previously segmented classes.
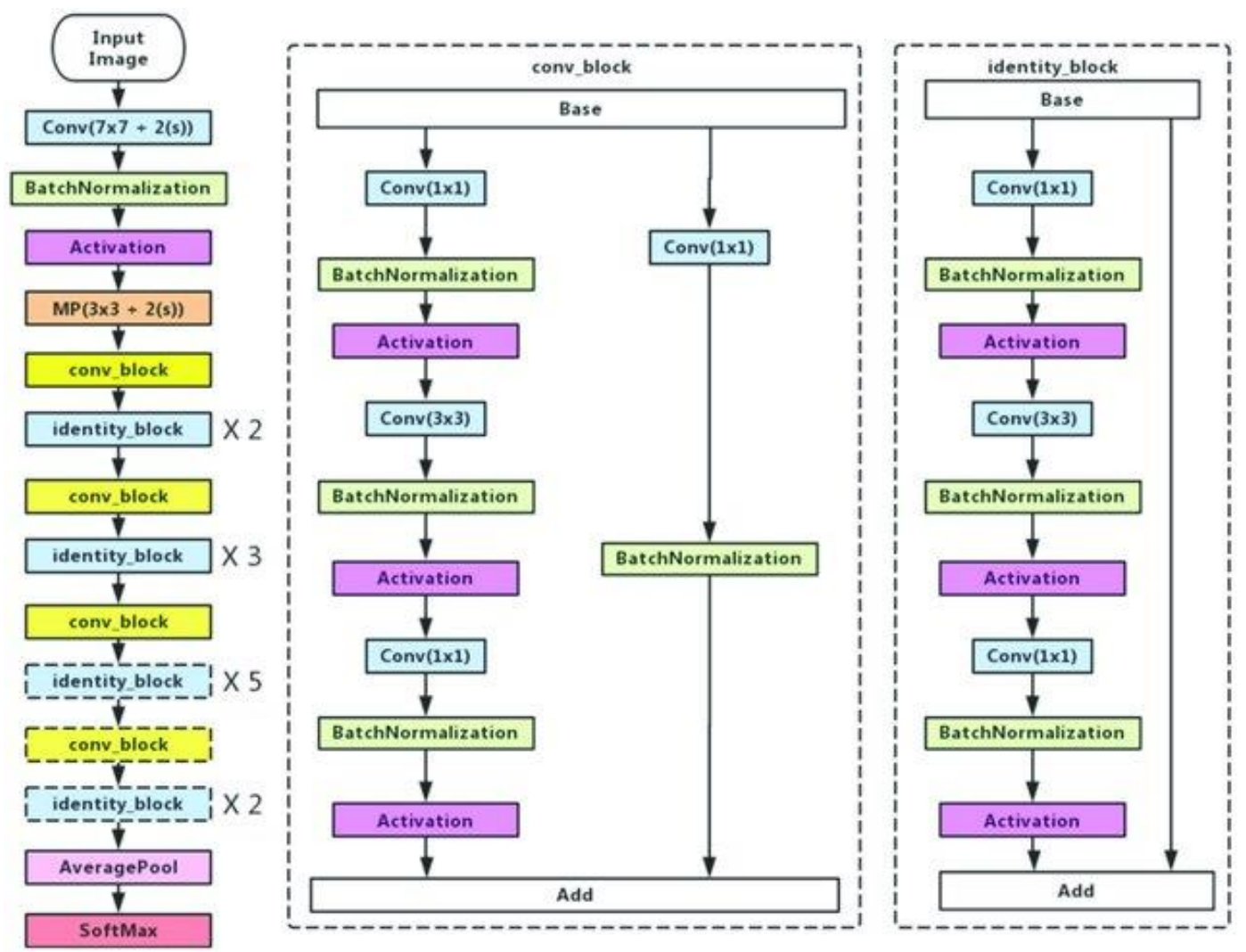

Fig. 1: Simplified visualization of the used ResNet-50 architecture (Ji et al. 2019).

Deep convolutional networks are an ideal solution for extracting features from image data, while stacking multiple layers improves prediction capabilities to some extent. Here, however, we encounter a known limitation that prevents the convergence of networks with dozens of layers: the problem of vanishing and exploding gradient. Adding additional layers to the model can increase the error value for the training set (Feng 2017). The ResNet-50 architecture consists of five stages, each with a convolutional block and an identity block, which is primarily used as a shortcut connection (He et al. 2015). The convolution block contains three convolution layers, each identity block operating with three convolution layers. The shortcut is used to skip one or more layers, e.g. from the first layer it is possible to skip to the third layer using the shortcut. 


\section{Summary of previous research}

Similar research can be found, for example, in Elgendi et al. (2020) who compared 17 available deep learning algorithms for easier, faster, and most importantly cheaper detection of COVID19 using chest radiography and the DarkNet-19 deep neural network. As a result, DarkNet-19 proved to be the optimal pre-trained network for detecting radiographic images of COVID-19 pneumonia with $94.28 \%$ accuracy in 5,854 X-rays available from the Kaggle database and another dataset from Vancouver Hospital. The input images of the network, showing posteroanterior and anteroposterior projection, were verified by radiologists with many years of experience. For validation, the networks were provided with a million different images and a balanced set of healthy individuals and those with pneumonia and COVID-19, with DarkNet19 showing the most appropriate balance between speed and reliability of detection. Further for comparison, e.g. Narin et al. (2020) report an accuracy of $98 \%$ using a convolutional network on a balanced dataset, Sethy and Behera (2020) report an accuracy of 93\% on a balanced dataset, Zhang et al. (2020) also report 93\%, but on an unbalanced dataset, Hemdam et al. (2020) 90\% on a balanced or Apostolopoulos and Bessiana (2020) 98\% on an unbalanced data sample.

The study by Elgendi et al. worked with various architectures in the MATLAB environment. Although the results were promising, the complete dataset included only 50 COVID-19 positive patients, so it was severely limited, with less confusion of infection with bacterial or viral pneumonia. Narin et al. as well as Sethy and Behera list ResNet50 as the most suitable network: this architecture is also used in our study. Sethy and Behera combined the ResNet50 and SVM convolutional network with histograms to achieve $93.4 \%$ accuracy in the validation set. Zhang et al. collected 100 chest X-rays in the GitHub database, of which 70 had confirmed COVID19 cases, thousands more with different pneumonia infections and healthy patients, but as a result, the model shows too high a percentage of false-positive findings. As well as other studies, they are aware of the need for subsequent validation of the collected clinical data. Hemdan et al. also worked with only a small dataset comprising 50 images, of which 25 were confirmed as COVID-19 positive. Apostopoulos and Bessiana had a larger database, including 224 images of COVID-19 positive patients. 


\section{Practical application of pneumonia detection model}

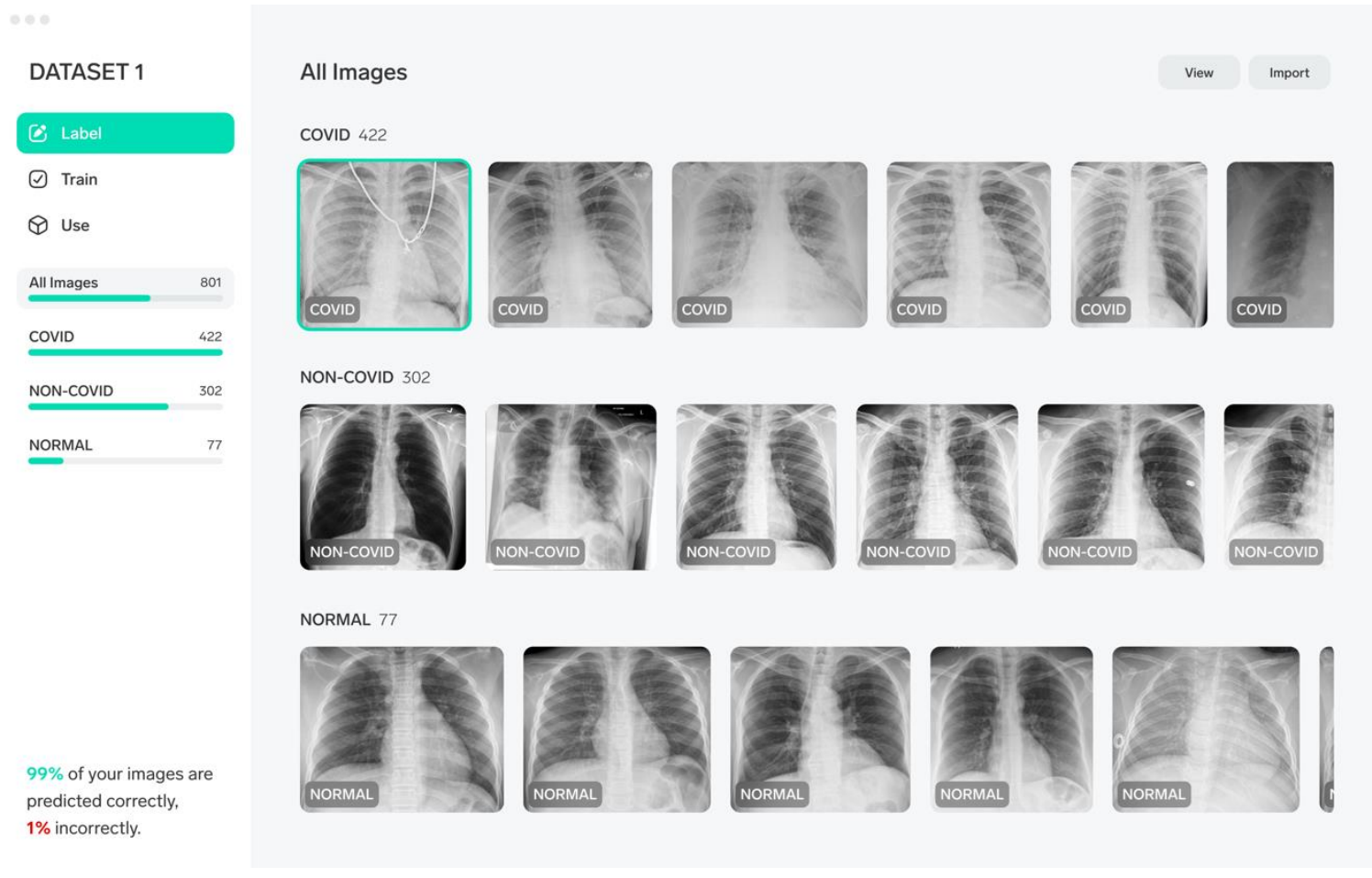

Fig. 2: Current visualization of the working environment for neural network training.

Dataset 1 comes from the open-source Mendeley Data repository. The data set was collected by authors from three different medical facilities in Uttar Pradesh and Rajasthan. 188 images (28 COVID-19, 83 with findings, 77 healthy images) were taken at King George's Medical University in Uttar Pradesh. 68 images of patients with COVID-19 were collected from the University of Medical Sciences in Uttar Pradesh. 543 X-rays (326 COVID-19, 217 with findings) come from Government Medical College in Rajasthan.

Dataset 2 was obtained from the Kaggle public data repository. Chest X-rays were selected from retrospective cohorts of pediatric patients aged one to five years from Women and Children's Medical Center in Guangzhou. All images were performed as part of routine clinical patient care. Prior to chest x-ray analysis, all images were first subjected to quality control and removal of any poor quality or illegible scans. The diagnoses were subsequently verified by two specialists. The data set was also checked by a third-party expert to take account of possible errors in the evaluation. The dataset consists of 2530 images with bacterial pneumonia, 1345 images with viral disease and 1341 images without findings. 
Dataset 3 is a collection of three large datasets that have been collected on the Kaggle datastore. Following the needs of our research, the individual classes were reduced to a simple distinction between images with and without pneumonia findings. The motivation of this dataset can be defined as a binary classification, where the input is an X-ray of the chest, while the output is a binary label indicating the absence or presence of a finding of pneumonia.

\begin{tabular}{|c|c|c|c|}
\hline Dataset & Type of classification & $\begin{array}{c}\text { Number of data for } \\
\text { each class }\end{array}$ & $\begin{array}{c}\text { Prediction accuracy on } \\
\text { a test set }\end{array}$ \\
\hline Dataset $1^{3}$ & Multi-class classification & $\begin{array}{c}\text { COVID-19 (422), } \\
\text { Non-COVID-19 (302), } \\
\text { Normal (77) }\end{array}$ & $99 \%$ \\
\hline Dataset $2^{4}$ & Multi-class classification & $\begin{array}{c}\text { Bacterial (2530), } \\
\text { Normal (1341), Viral } \\
(1345)\end{array}$ & $92 \%$ \\
\hline Dataset $3^{5}$ & Binary classification & $\begin{array}{c}\text { Normal (3293), } \\
\text { Pneumonia (8352) }\end{array}$ & $99 \%$ \\
\hline
\end{tabular}

Table 1: Predictive accuracy of the ResNet-50 neural network on individual datasets.

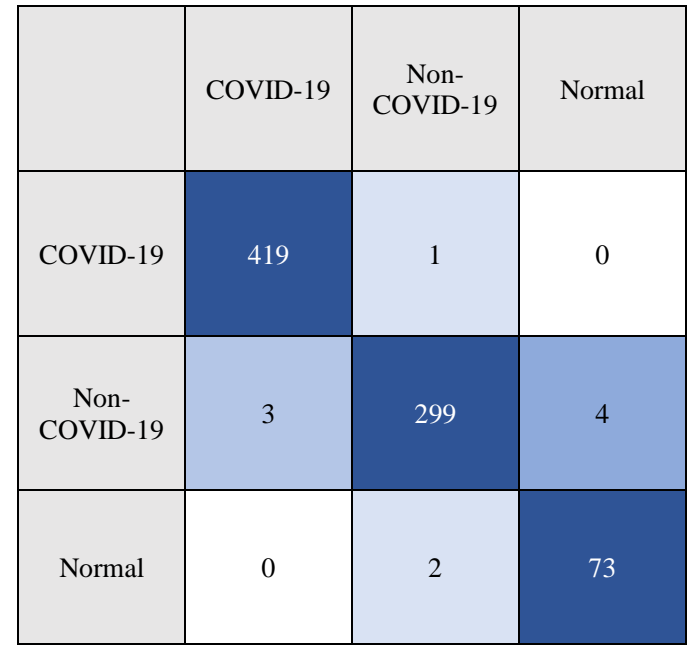

Table 2: Confusion matrix for dataset 1 .

\footnotetext{
${ }^{3}$ Dutta, M. K. (2021). COVID Chest X-Rays. 1. https://doi.org/10.17632/4n66brtp4j.1

4 Mooney, P. (b.r.). Chest X-Ray Images (Pneumonia). Retrieved 6 April 2021, from https://kaggle.com/paultimothymooney/chest-xray-pneumonia

${ }^{5}$ Kvak, D. (2021). Sběr RTG snímků plic z volně přístupných databází.
} 


\begin{tabular}{|c|c|c|c|c|}
\hline Class & Accuracy & Precision & Recall & F1 score \\
\hline COVID-19 & $99,5 \%$ & 1,0 & 0,99 & 1,0 \\
\hline Non-COVID-19 & $98,75 \%$ & 0,98 & 0,99 & 0,98 \\
\hline Normal & $99,25 \%$ & 0,97 & 0,95 & 0,96 \\
\hline
\end{tabular}

Table 3: Performance measure of multiclass classifier for dataset 1.

\section{Limitations of the used methodology}

Although radiological imaging is a widely available, as well as affordable method, due to the dangers of ionizing radiation from CT and X-ray equipment, it is not suitable for frequent use, and there are other contraindications to this procedure. It is necessary to consider imaging pregnant women, other complications can be caused by the presence of metal objects, the inability of the patient to take a deep full breath or also to adapt the equipment for too thin or obese patients (Uppot 2018).

The disadvantage of all similar studies, apart from the ongoing validation that awaits this research, may be the excessive technical complexity of projects (e.g. the use of MATLAB or Python). The primary goal of this study is therefore to provide a user-friendly environment suitable for inexperienced assessors under stress, and thus help early detection and faster action in patients with more severe stages of the disease. Imaginary barriers may include the impossibility of importing through the traditional DICOM imaging format used for the transmission of biomedical image data within PACS systems. This limitation can be circumvented by implementing the med2image or pydicom libraries.

\section{Discussion}

Chest radiography is a widely available and affordable tool for screening patients with symptoms of lower respiratory tract infection or suspected pneumonia. Automatic detection using X-rays can act as an early diagnosis of the disease; this is especially true in areas facing a shortage of trained radiologists. Based on preliminary results, we demonstrate that the use of deep learning methods, hence the ResNet-50 architecture, can be a functional solution for automatic feature extraction from X-rays related to the diagnosis of viral and bacterial pneumonia, or directly to the detection of diseases caused by COVID- 19 . 
However, it is necessary to investigate whether the extracted features segmented by a deep neural network represent reliable biomarkers that help detect lung infection. Follow-up research should focus on the sensitivity of imaging patients with mild symptoms: these symptoms may not be accurately imaged by X-rays or may not even be visible at all. It is also necessary to accept the fact that each medical or experimental workplace scans X-ray images in a different way, including image quality, angle or brightness. Although the research is conceived primarily as a source of initial estimation, we believe that using a sufficiently general dataset, this solution could present great promise for the future. While datasets often contain, for example, only one age group, our follow-up goal is to create a comprehensive database that can be used to generalize the findings.

\section{Acknowledgment}

We would like to take this opportunity to thank MUDr. Stanislav Kašik from the Radiodiagnostic Department of the Regional Hospital in Kolín, Ing. Dalibor Kačmár̆, Ph.D. from Microsoft and doc. Radim Burget, Ph.D. from the Faculty of Electrical Engineering and Communication VUT in Brno for their willingness and valuable advice. 


\section{References:}

Apostolopoulos, I. D., \& Bessiana, T. (2020). Covid-19: Automatic detection from X-Ray images utilizing Transfer Learning with Convolutional Neural Networks. Physical and Engineering Sciences in Medicine, 43(2), 635-640. https://doi.org/10.1007/s13246-020-008654

Du, Z. et al. (2021). Comparative cost-effectiveness of SARS-CoV-2 testing strategies in the USA: A modelling study. The Lancet Public Health, 6(3), e184-e191. https://doi.org/10.1016/S2468-2667(21)00002-5

Dutta, M. K. (2021). COVID Chest X-Rays. 1. https://doi.org/10.17632/4n66brtp4j.1

Elgendi, M. et al (2020). The Performance of Deep Neural Networks in Differentiating Chest X-Rays of COVID-19 Patients From Other Bacterial and Viral Pneumonias. Frontiers in Medicine, 7. https://doi.org/10.3389/fmed.2020.00550

Feng, V. (2017, July 17). An Overview of ResNet and its Variants. Medium. https://towardsdatascience.com/an-overview-of-resnet-and-its-variants-5281e2f56035

Gandhi, D. et al. (2020). Clinical Landscape of COVID-19 Testing: Difficult Choices. arXiv:2011.04202 [q-bio]. http://arxiv.org/abs/2011.04202

He, K. et al. (2015). Deep Residual Learning for Image Recognition. arXiv:1512.03385 [cs]. http://arxiv.org/abs/1512.03385

Hemdan, E. E.-D. et al. (2020). COVIDX-Net: A Framework of Deep Learning Classifiers to Diagnose COVID-19 in X-Ray Images. arXiv:2003.11055 [cs, eess]. http://arxiv.org/abs/2003.11055

Ji, Q. et al. (2019). Optimized Deep Convolutional Neural Networks for Identification of Macular Diseases from Optical Coherence Tomography Images. Algorithms, 12(3), 51. https://doi.org/10.3390/a12030051

Mina, M. J. (2021). Clarifying the evidence on SARS-CoV-2 antigen rapid tests in public health responses to COVID-19. The Lancet, O(0). https://doi.org/10.1016/S0140-6736(21)00425-6 
Mooney, P. (n.d.). Chest X-Ray Images (Pneumonia). Retrieved 6 April 2021, from https://kaggle.com/paultimothymooney/chest-xray-pneumonia

Narin, A. et al. (2020). Automatic Detection of Coronavirus Disease (COVID-19) Using X-ray Images and Deep Convolutional Neural Networks. arXiv:2003.10849 [cs, eess]. http://arxiv.org/abs/2003.10849

Papers with Code-ImageNet Benchmark (Image Classification). (b.r.). Retrieved 6 April 2021, from https://paperswithcode.com/sota/image-classification-on-imagenet

Rahman, T. et al. (2021). Exploring the effect of image enhancement techniques on COVID-19 detection using chest X-ray images. Computers in Biology and Medicine, 132, 104319. https://doi.org/10.1016/j.compbiomed.2021.104319

Sethy, P. K., \& Behera, S. K. (2020). Detection of Coronavirus Disease (COVID-19) Based on Deep Features. https://doi.org/10.20944/preprints202003.0300.v1

Uppot, R. N. (2018). Technical challenges of imaging \& image-guided interventions in obese patients. The British Journal of Radiology, 91(1089). https://doi.org/10.1259/bjr.20170931

Zhang, J. et al. (2020). Viral Pneumonia Screening on Chest X-ray Images Using ConfidenceAware Anomaly Detection. arXiv:2003.12338 [cs, eess]. http://arxiv.org/abs/2003.12338 